\title{
Intra-rater reliability and concurrent validity of quantified muscle testing for maximal knee extensors strength in men with myotonic dystrophy type
}

1.

Marie-Pier Roussel, ${ }^{\mathrm{a}, \mathrm{c} \text { and d }}$ Luc J. Hébert, ${ }^{\mathrm{e}, \mathrm{f}}$ and Elise Duchesne ${ }^{\mathrm{b}, \mathrm{c} \text { and } \mathrm{d} *}$

a) Département des sciences fondamentales, Université du Québec à Chicoutimi, Saguenay, Québec, Canada.

b) Département des sciences de la santé, Université du Québec à Chicoutimi, Saguenay, Québec, Canada.

c) Groupe de recherche interdisciplinaire sur les maladies neuromusculaires (GRIMN), Centre intégré universitaire de santé et de services sociaux du Saguenay-Lac-St-Jean, Saguenay, Québec, Canada.

d) Centre de recherche Charles-Le Moyne - Saguenay-Lac-Saint-Jean sur les innovations en santé, Saguenay, Québec, Canada.

e) Centre interdisciplinaire de recherche en réadaptation et intégration sociale (CIRRIS), Québec, Québec, Canada.

f) Départements de réadaptation et de radiologie-médecine nucléaire, Faculté de médecine, Université Laval, Québec, Québec, Canada.

Running title: DM1 muscle testing reliability and validity

*Corresponding author:

Elise Duchesne, pht, Ph.D.

Unité d'enseignement en physiothérapie, Département des sciences de la santé, Université du Québec à Chicoutimi

555, boul. de l'Université Chicoutimi, Québec, Canada G7H 2B1

Office: 418 545-5011 (\#6148); Fax: 418-545-5012

E-mail: elise1_duchesne@uqac.ca 


\begin{abstract}
BACKGROUND: Myotonic dystrophy type 1 (DM1) is the most prevalent degenerative neuromuscular disease in adults. Knee extensor (KE) maximal strength loss is a strong indicator of physical limitations in DM1. A reliable, precise and accessible maximal strength evaluation method needs to be validated for this slowly progressive disease.
\end{abstract}

OBJECTIVE: This paper aims to assess the intra-rater reliability, the standard error of measurement (SEM), the minimal detectable change (MDC), and the concurrent validity of quantified muscle testing (QMT) using a handheld dynamometer with a gold standard: the Biodex isokinetic device.

METHODS: Nineteen men with the adult form of DM1 participated in this study by attending 2 visits spaced by one week. The evaluation of KE muscle strength with QMT was completed on the first visit and the same QMT evaluation in addition to the maximal muscle strength evaluation using an isokinetic device were performed on the second visit.

RESULTS: The intra-rater reliability was excellent with an intraclass correlation coefficient (ICC) of 0.98 (0.96-0.99: 95\% confidence interval). SEM and MDC values were $1.05 \mathrm{Nm}$ and $2.92 \mathrm{Nm}$, respectively. Concurrent validity of QMT of KE muscle group with the Biodex was also excellent with a Spearman's correlation of $\rho=0.98$.

CONCLUSIONS: The excellent concurrent validity and intra-rater reliability, and the small SEM and MDC of the QMT make this test a method of choice, in either a clinical or research setting, to precisely evaluate muscle strength impairments of the KE in men with DM1.

Keywords: myotonic dystrophy type 1, muscle strength, quantified muscle testing, isokinetic device, intrarater reliability, concurrent validity 


\section{Introduction}

Myotonic dystrophy type 1 (DM1) is the most prevalent degenerative hereditary myopathy in adults and is caused by an abnormal CTG triplet repetition on the myotonic dystrophy protein kinase (DMPK) gene [1]. The age of onset of the disease along with its severity are mildly correlated with the number of CTG repetitions [2]. In general, the severity of the symptoms is also correlated with the age of onset of the disease. The disease is classified into five different phenotypes according to the age of onset: congenital, infantile, juvenile, adult or classic and late [3]. DM1 is a multisystemic disease, which affects cardiovascular, endocrine, neurological and musculoskeletal systems, to name a few [4]. Neurological affections can include cognitive impairments, daytime sleepiness and apathy [4-6]. Skeletal muscles are notably affected and patients with the disease experience slowly progressive muscle atrophy and weakness [4, 7]. In the adult form, it has been demonstrated that affected people lose 24.5 to $52.8 \%$ of their maximal strength on a 9-year period depending on the muscle group evaluated [8]. Furthermore, it has been reported that the disease affects skeletal muscles differently in men and women[8-10]. Petitclerc et al. have demonstrated that muscle weakness in knee extension and ankle dorsiflexion is a very good indicator of physical limitations in people with the adult or late phenotype of DM1 [11]. They also showed that knee extensors (KE) muscle strength was the main explanatory factor of performance variations in the timed up and go test [11]. Lower extremity weakness is also one of the main predictors of disruption in participation of the affected individuals $[12,13]$. It is therefore important that lower extremity maximal strength, namely for the $\mathrm{KE}$ and the ankle dorsiflexors, can be robustly and finely measured in this population. These precise measurements will not only be useful to follow the disease's progression, but also to verify the efficiency of treatments in the context of clinical follow-ups or clinical trials timeframes. Intra-rater reliability and the standard error of measurement (SEM) for the ankle dorsiflexors maximal strength using a handheld dynamometer have already been calculated for this population [14]. However, such data do not yet exist for the KE muscle group, another important clinical indicator of physical limitations in DM1 adult and late phenotypes. 
One method that is commonly used in DM1 to measure muscle strength is manual muscle testing (MMT) as it is incorporated in the muscle impairment rating scale (MIRS), a tool designed to rate the severity of muscle impairments in DM1 [15]. However, MMT has been demonstrated to be unreliable in DM1 as well as in other populations $[14,16,17]$. Indeed, it has been shown that for the same muscle strength expressed in Nm, DM1 participants could be classified in multiple MMT scores [16]. More importantly, DM1 participants that were considered to have no muscle impairments according to the MMT, showed significantly lower maximal strength than their predicted values [16]. In addition, it has been shown that to detect KE strength loss with MMT, a person with DM1 must have lost on average $45.2 \%$ of its maximal strength [18]. A more discriminant and precise method to evaluate maximal muscle strength therefore needs to be validated in this population, not only for research purposes but for clinical practice as well. Isokinetic devices such as the Biodex system 4 (Biodex Medical Systems, Shirley, NY, USA) are considered to be the gold standard for maximal muscle strength evaluation. However, these devices have many inconveniences, namely in a clinical setting: they are very expensive, cannot be moved easily, are quite time consuming to use, and some testing positions are difficult to adopt by DM1 patients [19]. A compromise between the affordability of MMT and the high precision and good reliability and validity of the isokinetic devices resides with quantitative muscle testing (QMT) techniques using a handheld dynamometer [19]. Handheld dynamometry is widely used in physiotherapy practice, however, there is a lack of standardisation in the methods across studies [20]. In order to carry out an adequate handheld dynamometry protocol, some elements must be respected to diminish biases. First of all, make tests have been shown to be more reliable [21] and less likely to cause injury than break tests [22]. Next, positions that eliminate gravity for the movement of interest avoid having to consider the weight of the limb in the calculation of muscle torques [23]. Thereafter, the main agonist muscles should be placed at mid-length to allow the generation of maximal strength during contraction [24]. Lastly, whenever possible, the involvement of the evaluator in providing the physical resistance should be minimized. The handheld dynamometry QMT technique presented in this study met all those criteria [23]. 
The main objective of this paper was to evaluate the reliability and validity of the evaluation of the maximal isometric muscle strength (MIMS) of the KE using the QMT technique in men with the adult form of DM1. More specifically, this study aims to: 1) evaluate the intra-rater reliability along with the SEM and the minimal detectable change (MDC) of the QMT technique and 2) to evaluate the concurrent validity of the QMT technique with a Biodex isokinetic device.

\section{Materials and methods}

Men with the adult form of DM1 aged between 30 and 65 years old were recruited from the neuromuscular clinic of Jonquière, Québec, Canada. DM1 diagnosis had to be confirmed by genetic analysis. To limit heterogeneity in the group, recruitment was restrained to men. Participants had to be able to walk without aids, give their informed consent, reside in the Saguenay-Lac-Saint-Jean region and have no contraindications to maximal resistance testing. The study was approved by the Ethics Review Board of the Centre intégré universitaire de santé et de services sociaux (CIUSSS) of Saguenay-Lac-Saint-Jean (Québec, Canada) and all participants gave their written informed consent.

The evaluations were done on two separate visits. In the first visit, participants completed QMT for the KE on both legs. One week later, the participants did both: 1) QMT for the KE on both legs again and 2) isometric muscle testing of the right $\mathrm{KE}$ on the Biodex isokinetic device with a mandatory 30 minutes sitting rest between the two different testing methods. The participants were randomly assigned to either complete the QMT first or the Biodex. The weight of all participants was measured using the InBody 570 scale (InBody, Ottawa, Canada). All tests were administered by the same, previously trained, physical therapist (M-P R).

\subsection{Quantified muscle testing}

The QMT protocol used in this study to evaluate the MIMS was developed by Hébert et al. to ensure the best reproducibility of handheld dynamometry evaluations [23]. The handheld dynamometer used was the MEDup $^{\mathrm{TM}}$ linear electronic handheld dynamometer (Atlas medic, Québec, Canada). Participants were 
seated with their hip and knee flexed at a 90-degree angle (Figure 1-A). A pen mark was made on the patient's leg $10 \mathrm{~cm}$ above the inferior edge of the lateral malleolus as a position indicator for the proximal edge of the dynamometer. The dynamometer was positioned perpendicular with the leg and secured in place with a certified strap. Participants were asked to push gradually against the dynamometer until they reached their maximal strength and to hold the contraction until they were told to release. During the test, patients were given a vigorous standardized encouragement that lasted 10 seconds to ensure that peak force was reached. The encouragements started with a neutral tone and gradually progressed in intensity, the evaluator began with "go ahead, push” and continued with the use of the words "push”, and "stronger” alternatively and the participant's name to end with "and now release” once the 10 seconds were completed. Participants were allowed to stabilize themselves by holding the edge of the table with both hands, with their elbows in full extension to avoid them to bend forward or backward during the tests. At least two trials were done: if the trials had more than $10 \%$ difference, a third or fourth trial was done as needed. The mean of the two closest trials for each leg was kept for analysis. This procedure was chosen to ensure the result was as close as possible to the real maximal strength and minimize possible errors during the test (compensations, submaximal contractions, etc.). Participants were given a 30 second rest between trials. The lever arm length was calculated as being the perpendicular distance between the knee joint line and the pen mark to which half the width of the dynamometer's cushion was added. The knee joint line was chosen as the reference point to the knee to allow better intra and inter-rater reproducibility [23].

\subsection{Isokinetic device}

The isokinetic device used was the Biodex isokinetic dynamometer (Biodex Medical Systems, Shirley (NY), USA). Participants were seated according to the manufacturer's instructions with all belts fastened and hip and knee at 90-degree angles (Figure 1-B). The center of the isokinetic device was positioned to be aligned with the right knee's joint center of rotation: at the inferior part of the lateral condyle. To evaluate the MIMS, the isokinetic device was programmed to record three isometric 10-second trials with 30 seconds 
of rest between trials. The participants were given the same vigorous standardized encouragements during the isokinetic device testing as during the QMT.

\subsection{Data analysis}

QMT intra-rater reliability was calculated by comparing the average measure of the two closest trials of both legs (4 measurements) from week 1 to the average measure from week 2. Intraclass correlations coefficients (ICC) with a 95\% confidence interval and coefficient of determination $\left(\mathrm{R}^{2}\right)$ were used to assess reliability. Bland and Altman plots were also used for further analysis purposes and confirm adequate reproducibility between week 1 and week 2. In each plot, the mean of the difference along with the 95\% confidence interval bounds are presented. A standard error of the measurement (SEM) was also calculated from this data. The minimal detectable change (MDC) with a 95\% confidence interval was calculated using the formula MDC $=1.96 \times \mathrm{SEM} \times \sqrt{ } 2$, where 1.96 is derived from the $95 \%$ confidence interval. This value was also converted in percentage compared to the average of the group, where MDC $\%=$ (MDC/average) $* 100$.

Concurrent validity between the QMT and the Biodex isokinetic device was calculated using the average of the closest two trials of the isokinetic device testing (right leg only) compared to the closest two trials of the right leg with QMT technique, both obtained on week two. Spearman's correlation and $\mathrm{R}^{2}$ were used to assess correlation between the two evaluation methods. Bland and Altman plots were also used to evaluate the agreement between the two different measurements techniques used.

\section{Results}

\subsection{Participants}

A convenience sample of 21 male participants with DM1 was recruited. One participant dropped out before his first visit and another was excluded because he was not able to understand the instructions related to maximal muscle strength evaluations (QMT and isokinetic device). If possible, the participants were evaluated on the same time of day for both evaluations to avoid different levels of fatigue: 14/19 were 
evaluated at the same time of day and 5/19 were evaluated at different times due to work schedule constrains. Out of those 5 patients, none were evaluated with a morning/evening extreme. Participant's characteristics are found in Table 1. Patients are presented according to their KE maximal strength obtained during Biodex testing.

\subsection{Intra-rater reliability of the QMT}

An excellent ICC (ICC > 0.90) [25] of 0.98 with a 95\% confidence interval ranging from 0.96 to 0.99 was obtained by comparing the QMT results of week 1 and week 2 (Table 2). The SEM obtained was fairly low (1.05 Nm) along with a low MDC (2.92 Nm) and MDC\% (2.69\%). As seen on the Bland and Altman plot of the Figure 2, the mean of the difference between week 1 and 2 is very small (3.37 Nm), meaning that repeating this method would lead to similar results over time. A paired t-test done between week 1 and 2 (Table 1, intra-rater reliability rows) confirms this with $\mathrm{p}=0.16$.

\subsection{Concurrent validity between the QMT and the isokinetic device}

A very high correlation was found between the QMT and Biodex isokinetic device for the evaluation of MIMS in men with DM1 $\left(\mathrm{R}^{2}=0.94\right.$ and $\left.\rho=0.98\right)$ (Table 3). However, the mean difference between the methods in accordance to the Bland and Altman plot (Figure 3) is $23.67 \mathrm{Nm}$ ( $\mathrm{p}<0.001$ ), which means that the QMT technique on average provides lower values of MIMS than the isokinetic device.

\section{Discussion}

The aim of this study was to determine the intra-rater reliability, the SEM, the MDC and the concurrent validity with the isokinetic device of the QMT technique in men with the adult form of DM1. The intrarater reliability was found to be excellent (ICC > 0.90) [25] with an ICC of 0.98 . The $95 \%$ confidence interval was also very high, ranging from 0.96 to 0.99 and thus remains excellent throughout its range. The SEM and MDC were also very low at $1.05 \mathrm{Nm}$ and $2.92 \mathrm{Nm}$, respectively. This means that a change of $1.05 \mathrm{Nm}$ can be considered into the error of the measurement but any change greater than $2.92 \mathrm{Nm}$ can be considered as the result of the patient's maximal strength variation. The SEM obtained for the MIMS of 
$\mathrm{KE}$ is comparable to the one previously published for ankle dorsiflexion in DM1, which is $1.0 \mathrm{Nm}$ [14]. Considering that the strength of the $\mathrm{KE}$ is much higher than the ankle dorsiflexors, this later finding confirms that, when using an appropriate QMT method, the capacity to resist of the evaluator does not impact at all on the measurement error. The same QMT protocol was used in healthy children aged from 13 to 17.5 years old: while the SEM is comparable for ankle dorsiflexion (0.5 Nm for girls and $1.0 \mathrm{Nm}$ for boys), the SEM for knee extension was higher (3.8 Nm for girls and 4.5 for boys) than the one we obtained [23]. This means that despite the possible difficulties in assessing maximal strength in DM1 related to cognitive impairment and/or apathy, QMT for KE and ankle dorsiflexors remain highly reliable and sensitive to change in this population. These results are quite encouraging and useful for clinicians that have to finely monitor their patient's strength and evaluate the efficacy of their interventions in this slowly progressive disease. Specifically, our results showed that a variation of $2.92 \mathrm{Nm}$ (5.9-6.1\% of the maximal KE strength) in KE muscle strength evaluated with QMT technique is clinically significant, in comparison with MMT technique where $45.2 \%$ of maximal strength loss is required to be able to conclude about the presence of KE muscle impairments [18].

A strength of the present results is that many sources of biases have been controlled for. The physiotherapist in charge of administrating all the tests was trained with the QMT and isokinetic device evaluation techniques. Parameters previously mentioned, such as agonist muscle length and gravity were controlled for. Another important factor that was controlled was the encouragements provided to the participants. While it is important that the participants are adequately stimulated throughout all populations, it is particularly important in DM1 since apathy is very frequent in this disease [6]. All of these factors, as well as a highly standardized technique should be respected while evaluating maximal muscle strength. And finally, the use of a high-quality handheld dynamometer both in terms of load cell precision and excellent equipment ergonomics has also contributed to obtain precise and reproducible measure.

Regarding concurrent validity, the Spearman's correlation and $\mathrm{R}^{2}$ between the QMT technique and the isokinetic device are also very high $\left(\mathrm{R}^{2}=0.94\right.$ and $\left.\rho=0.98\right)$, meaning that the QMT compares to a well- 
established test considered as the gold standard, the Biodex. In other words, the higher the individual scores on the QMT, the higher their score on the Biodex and likewise, the lower the score on QMT, the lower the score on the Biodex. However, according to the Bland an Altman plot, we have found that there was on average a difference of 23.7 Nm between the QMT and Biodex method. The plot also shows that the stronger the patient, the more likely there is to be higher values obtained with the Biodex. There are different methodological factors to consider explaining this systematic difference that are discussed in the next section.

\subsection{Study limitations}

One important difference between the QMT and the Biodex comes from the measurement of the lever arm. In the QMT, in order to assure a better reproducibility, the knee joint line was chosen as the anatomical reference point for the axis of knee joint rotation [23], while with the isokinetic device, it was the inferior part of the lateral femoral condyle. While these two anatomical structures are very close, the lateral condyle of the knee is slightly above the knee joint line, giving a longer lever arm during the isokinetic device testing, thus leading to the calculation of higher moments of KE muscle strength. The second difference between the two evaluation methods would be the trunk stabilization. For the isokinetic device, the participants are tightly strapped to the chair, giving them the most possible stable position (Figure 1-B). In the QMT, participants are simply seated on an evaluation table (Figure 1-A) and they had to stabilize themselves. This double task could lead to submaximal contractions of the KE in this population known to have cognitive impairments. In line with this, it has already been shown that double tasks negatively influence gait patterns in DM1 [26]. Also, during the QMT evaluation, we cannot exclude that the stabilization of patients by holding the front of the examination table may have resulted in a slight hip flexion, leading to a reduced length of the rectus femoris of the quadriceps. Being one of the main agonists of knee extension, a change in length of the rectus femoris in favor of a reduction of muscle length may explain some of the strength loss as the maximal strength of each muscle depends on its contraction length where in general mid-length will provide the greater muscle strength production [24]. 


\section{Conclusion}

Precisely measuring KE muscle strength in DM1 is fundamental as this muscle group is essential to maintain adequate mobility and reduce as much as possible physical limitations in this population. Also, in this slowly progressive disease, it is even more important to use a measure that is sensitive with a very small error in order to detect any short-term changes of muscle strength. Next, as it is easy and important to train the KE, the QMT method would allow clinicians to rapidly verify if the provided training is successful or not. The current findings are encouraging, as QMT has shown excellent intra-rater reliability, a very small error of measurement, and a good concurrent validity with the Biodex meaning that clinicians could use this method to finely monitor the muscle function of their DM1 patients with confidence. Moreover, the QMT technique proposed is user friendly, versatile, affordable and portable, and would therefore be an excellent method to evaluate maximal muscle KE strength in subjects with DM1.

\section{Acknowledgements}

The authors would like to thank all the participants for the time they have taken to complete this study. We would like to thank Dre Cynthia Gagnon for her support in the completion of this project by graciously lending services of the Groupe de recherche interdisciplinaire sur les maladies neuromusculaires (GRIMN). We would also like to thank Hélène Simard for her work to recruit all the participants in this study along with Dr Djamal Brebiche and Isabelle Côté for their help with the statistical analyses. We will also thank the Université du Québec à Chicoutimi for the access to the necessary equipment and the evaluation rooms. This project was funded by the Fondation du grand défi Pierre Lavoie. Marie-Pier Roussel also has a doctoral studies grant from the Fonds de recherche du Québec-Santé. The authors have no conflicts of interest to disclose. 


\section{References}

[1] Theadom A, Rodrigues M, Roxburgh R, Balalla S, Higgins C, Bhattacharjee R, et al. Prevalence of muscular dystrophies: a systematic literature review. Neuroepidemiology. 2014;43(3-4):259-68.

[2] Harley HG, Rundle SA, MacMillan JC, Myring J, Brook JD, Crow S, et al. Size of the unstable CTG repeat sequence in relation to phenotype and parental transmission in myotonic dystrophy. Am J Hum Genet. 1993;52(6):1164-74.

[3] De Antonio M, Dogan C, Hamroun D, Mati M, Zerrouki S, Eymard B, et al. Unravelling the myotonic dystrophy type 1 clinical spectrum: A systematic registry-based study with implications for disease classification. Rev Neurol (Paris). 2016;172(10):572-80.

[4] Harper PS. Myotonic dystrophy. 3rd ed. London: WB Saunders; 2001.

[5] Gallais B, Gagnon C, Mathieu J, Richer L. Cognitive decline over time in adults with myotonic dystrophy type 1: A 9-year longitudinal study. Neuromuscul Disord. 2017;27(1):61-72.

[6] Gallais B, Montreuil M, Gargiulo M, Eymard B, Gagnon C, Laberge L. Prevalence and correlates of apathy in myotonic dystrophy type 1. BMC Neurol. 2015;15:148.

[7] Tollbäck A, Eriksson S, Wredenberg A, Jenner G, Vargas R, Borg K, et al. Effects of high resistance training in patients with myotonic dystrophy. Scandinavian Journal Of Rehabilitation Medicine. 1999;31(1):9-16.

[8] Gagnon C, Petitclerc E, Kierkegaard M, Mathieu J, Duchesne E, Hebert L. A 9-year followup study of quantitative muscle strength changes in myotonic dystrophy type 1. J Neurol. 2018.

[9] Dogan C, De Antonio M, Hamroun D, Varet H, Fabbro M, Rougier F, et al. Gender as a Modifying Factor Influencing Myotonic Dystrophy Type 1 Phenotype Severity and Mortality: A Nationwide Multiple Databases Cross-Sectional Observational Study. PLoS One. 2016;11(2):e0148264.

[10] Raymond K, Levasseur M, Mathieu J, Desrosiers J, Gagnon C. A 9-year follow-up study of the natural progression of upper limb performance in myotonic dystrophy type 1: A similar decline for phenotypes but not for gender. Neuromuscul Disord. 2017;27(7):673-82.

[11] Petitclerc E, Hebert U, Mathieu J, Desrosiers J, Gagnon C. Relationships between Lower Limb Muscle Strength Impairments and Physical Limitations in DM1. J Neuromuscul Dis. 2018;5(2):215-24.

[12] Gagnon C, Mathieu J, Jean S, Laberge L, Perron M, Veillette S, et al. Predictors of disrupted social participation in myotonic dystrophy type 1. Arch Phys Med Rehabil. 2008;89(7):1246-55.

[13] Gagnon C, Mathieu J, Noreau L. Life habits in myotonic dystrophy type 1. J Rehabil Med. 2007;39(7):560-6.

[14] Hebert $\amalg$, Remec JF, Saulnier J, Vial C, Puymirat J. The use of muscle strength assessed with handheld dynamometers as a non-invasive biological marker in myotonic dystrophy type 1 patients: a multicenter study. BMC Musculoskelet Disord. 2010;11:72.

[15] Mathieu J, Boivin H, Meunier D, Gaudreault M, Begin P. Assessment of a disease-specific muscular impairment rating scale in myotonic dystrophy. Neurology. 2001;56(3):336-40.

[16] Petitclerc E, Hebert LJ, Mathieu J, Desrosiers J, Gagnon C. Lower limb muscle strength impairment in late-onset and adult myotonic dystrophy type 1 phenotypes. Muscle Nerve. 2017;56(1):57-63. 
[17] Noreau L, Vachon J. Comparison of three methods to assess muscular strength in individuals with spinal cord injury. Spinal Cord. 1998;36(10):716-23.

[18] Petitclerc E, Hebert LJ, Desrosiers J, Mathieu J, C G, editors. Relationship between lower limb muscle strength impairments and physical function in adult and late onset DM1 phenotypes. 10th International Myotonic Dystrophy Consortium Meeting; 2015 June 10 2015; Paris.

[19] Aufsesser P, Horvat M, Croce R. A Critical Examination of Selected Hand-Held Dynamometers to Assess Isometric Muscle Strength. Adapted Physical Activity Quarterly. 1996;13(2):153-65.

[20] Petitclerc E, Hebert LJ, Desrosiers J, Gagnon C. Lower limb muscle impairment in myotonic dystrophy type 1: the need for better guidelines. Muscle Nerve. 2015;51(4):473-8.

[21] Bohannon RW. Intertester reliability of hand-held dynamometry: a concise summary of published research. Percept Mot Skills. 1999;88(3 Pt 1):899-902.

[22] Seagraves FE, Horvat M. Comparison of Isometric Test Procedures to Assess Muscular Strength in Elementary School Girls. Pediatric Exercise Science. 1995;7(1):61-8.

[23] Hebert $\amalg$, Maltais DB, Lepage C, Saulnier J, Crete M, Perron M. Isometric muscle strength in youth assessed by hand-held dynamometry: a feasibility, reliability, and validity study. Pediatr Phys Ther. 2011;23(3):289-99.

[24] Brown L. Isokinetics in Human Performance2000.

[25] Koo TK, Li MY. A Guideline of Selecting and Reporting Intraclass Correlation Coefficients for Reliability Research. J Chiropr Med. 2016;15(2):155-63.

[26] Radovanovic S, Peric S, Savic-Pavicevic D, Dobricic V, Pesovic J, Kostic V, et al. Comparison of temporal and stride characteristics in myotonic dystrophies type 1 and 2 during dual-task walking. Gait Posture. 2016;44:194-9. 
Tables

Table 1:

Participant's characteristics and maximal muscle strength of their KE.

\begin{tabular}{|c|c|c|c|c|c|c|c|c|c|}
\hline \multirow[b]{2}{*}{$\begin{array}{l}\text { Patient } \\
\text { number }\end{array}$} & \multirow[b]{2}{*}{$\begin{array}{l}\text { Age } \\
\text { Years }\end{array}$} & \multirow[b]{2}{*}{$\begin{array}{c}\text { Age of } \\
\text { diagnosis } \\
\text { Years } \\
\end{array}$} & \multirow[b]{2}{*}{$\begin{array}{c}\text { Weight } \\
\mathrm{kg}\end{array}$} & \multirow[b]{2}{*}{$\begin{array}{c}\text { Height } \\
\mathrm{cm}\end{array}$} & \multirow[b]{2}{*}{$\begin{array}{c}\text { CTG } \\
\mathrm{n} \\
\end{array}$} & \multicolumn{2}{|c|}{$\begin{array}{l}\text { Values used for the intra-rater } \\
\text { reliability }\end{array}$} & \multicolumn{2}{|c|}{$\begin{array}{l}\text { Values used for the concurrent } \\
\text { validity }\end{array}$} \\
\hline & & & & & & $\begin{array}{c}\text { QMT * } \\
\text { week } 1 \\
\text { Nm }\end{array}$ & $\begin{array}{c}\text { QMT * } \\
\text { week } 2 \\
\text { Nm }\end{array}$ & $\begin{array}{c}\text { QMT right } \\
\text { leg }^{\ddagger} \\
\text { week } 2 \\
\text { Nm }\end{array}$ & $\begin{array}{c}\text { Isokinetic } \\
\text { device right } \\
\text { leg }^{\ddagger} \\
\text { week } 2 \\
\text { Nm }\end{array}$ \\
\hline 1 & 40 & 16 & 42.9 & 178 & 600 & 9.40 & 8.82 & 5.84 & 7.15 \\
\hline 2 & 51 & 27 & 71.1 & 173 & 1300 & 24.83 & 27.98 & 23.21 & 23.35 \\
\hline 3 & 37 & 32 & 56.7 & 170 & 500 & 40.45 & 40.87 & 37.16 & 36.55 \\
\hline 4 & 56 & 22 & 65.3 & 176 & 1000 & 76.08 & 77.95 & 70.24 & 73.15 \\
\hline 5 & 35 & 18 & 65.8 & 169 & 800 & 90.21 & 83.06 & 79.55 & 99.85 \\
\hline 6 & 54 & 20 & 66.5 & 163 & 1200 & 97.57 & 82.62 & 78.55 & 103.20 \\
\hline 7 & 48 & 35 & 71.2 & 172 & 250 & 107.44 & 95.70 & 89.13 & 113.25 \\
\hline 8 & 57 & 52 & 68.8 & 170 & 400 & 89.45 & 98.13 & 91.92 & 117.00 \\
\hline 9 & 36 & 29 & 51.0 & 169 & 400 & 90.76 & 87.79 & 89.61 & 118.70 \\
\hline 10 & 37 & 34 & 61.4 & 174 & 350 & 127.75 & 122.13 & 123.84 & 127.45 \\
\hline 11 & 60 & 39 & 70.7 & 167 & 400 & 98.23 & 114.99 & 119.87 & 144.20 \\
\hline 12 & 41 & 6 & 95.4 & 170 & 400 & 156.40 & 116.85 & 106.78 & 160.40 \\
\hline 13 & 61 & 55 & 99.0 & 170 & 80 & 132.80 & 131.87 & 136.40 & 170.65 \\
\hline 14 & 35 & 32 & 70.1 & 177 & 80 & 156.32 & 152.76 & 144.63 & 173.55 \\
\hline 15 & 41 & 28 & 109.9 & 172 & 400 & 135.58 & 126.82 & 134.07 & 185.75 \\
\hline 16 & 61 & 53 & 76.6 & 162 & 65 & 141.81 & 145.92 & 148.87 & 192.15 \\
\hline 17 & 51 & 44 & 92.7 & 168 & 70 & 179.17 & 190.23 & 197.18 & 198.70 \\
\hline 18 & 40 & 26 & 100.6 & 176 & 150 & 162.61 & 160.96 & 161.87 & 203.80 \\
\hline 19 & 32 & 25 & 114.8 & 182 & 250 & 175.59 & 162.96 & 165.29 & 204.95 \\
\hline $\begin{array}{l}\text { Mean } \\
(\mathrm{SD})\end{array}$ & $\begin{array}{l}46 \\
(10)\end{array}$ & $\begin{array}{c}32 \\
(13)\end{array}$ & $\begin{array}{l}76 \\
(20)\end{array}$ & $\begin{array}{l}172 \\
(6)\end{array}$ & $\begin{array}{c}458 \\
(372)\end{array}$ & $\begin{array}{l}110.13 \\
(49.16)\end{array}$ & $\begin{array}{l}106.76 \\
(47.62)\end{array}$ & $\begin{array}{l}105.47 \\
(50.33)\end{array}$ & $\begin{array}{l}129.15 \\
(61.50)\end{array}$ \\
\hline
\end{tabular}

Table legend: *Average of the two closest values of right and left leg (4 measurements). ${ }^{\ddagger}$ Average of the two closest values of right leg (2 measurements). 
Table 2:

Intra-rater reliability, SEM and MDC of the evaluation of maximal muscle strength of the KE muscle groups at two time points separated by one week

\begin{tabular}{lcccccc}
\hline $\begin{array}{l}\text { QMT of KE } \\
\text { (week 1 vs week 2) }\end{array}$ & $\mathrm{R}^{2}$ & ICC & $\begin{array}{c}\text { 95\% confidence } \\
\text { interval }\end{array}$ & SEM & MDC & MDC\% \\
\cline { 2 - 7 } & 0.94 & 0.98 & $0.96-0.99$ & $1.05 \mathrm{Nm}$ & $2.92 \mathrm{Nm}$ & $2.69 \%$ \\
\hline
\end{tabular}


Table 3:

Concurrent validity of QMT technique with an isokinetic device for the evaluation of maximal muscle strength of $\mathrm{KE}$

\begin{tabular}{lcc}
\hline Concurrent validity between & $\mathrm{R}^{2}$ & Spearman's $(\rho)$ \\
\cline { 2 - 2 } the QMT and the isokinetic & 0.94 & 0.98 \\
device for the KE & & $(\mathrm{p}<0.001)$ \\
\hline
\end{tabular}


Figure legends

Figure $1 \mathrm{~A}$ and B: Illustrations depicting the two different evaluation methods. A: The QMT technique using a handheld dynamometer. B: The isokinetic device technique. All line art was simplified from photographs to facilitate comprehension of the setup.

Figure 2: Bland Altman plot of the difference in KE maximal strength between week 1 and 2 using QTM for each participant $(\mathrm{p}=0.16)$. The upper and lower limits represent the $95 \%$ confidence interval. Results are presented in $\mathrm{Nm}$.

Figure 3: Bland Altman plot of the difference between the handheld dynamometry and the isokinetic device for each participant $(\mathrm{p}<0.001)$. The upper and lower limits represent the 95\% confidence interval. Results are presented in $\mathrm{Nm}$. 
Figures

Figure $1 \mathrm{~A}$ and $\mathrm{B}$

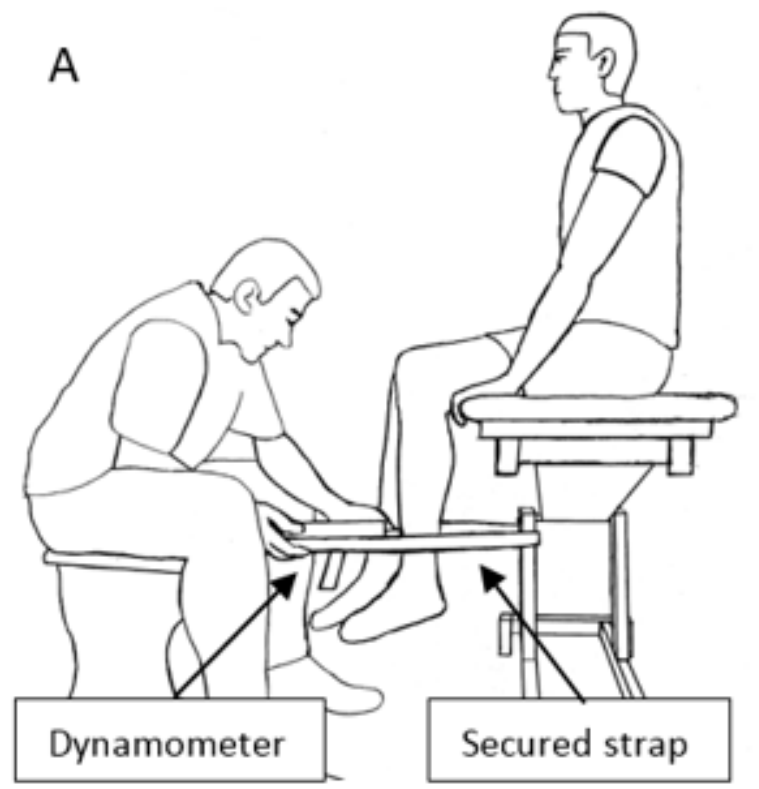

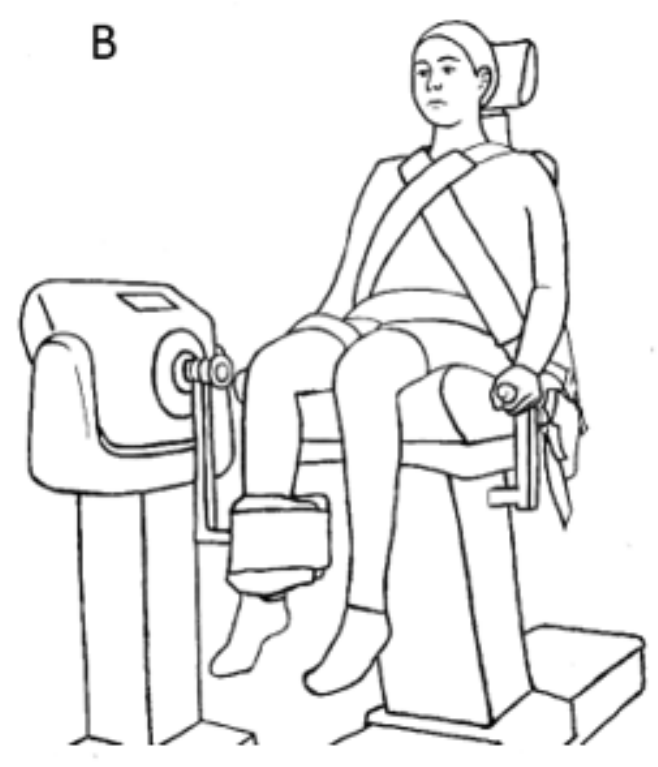

Figure 2 


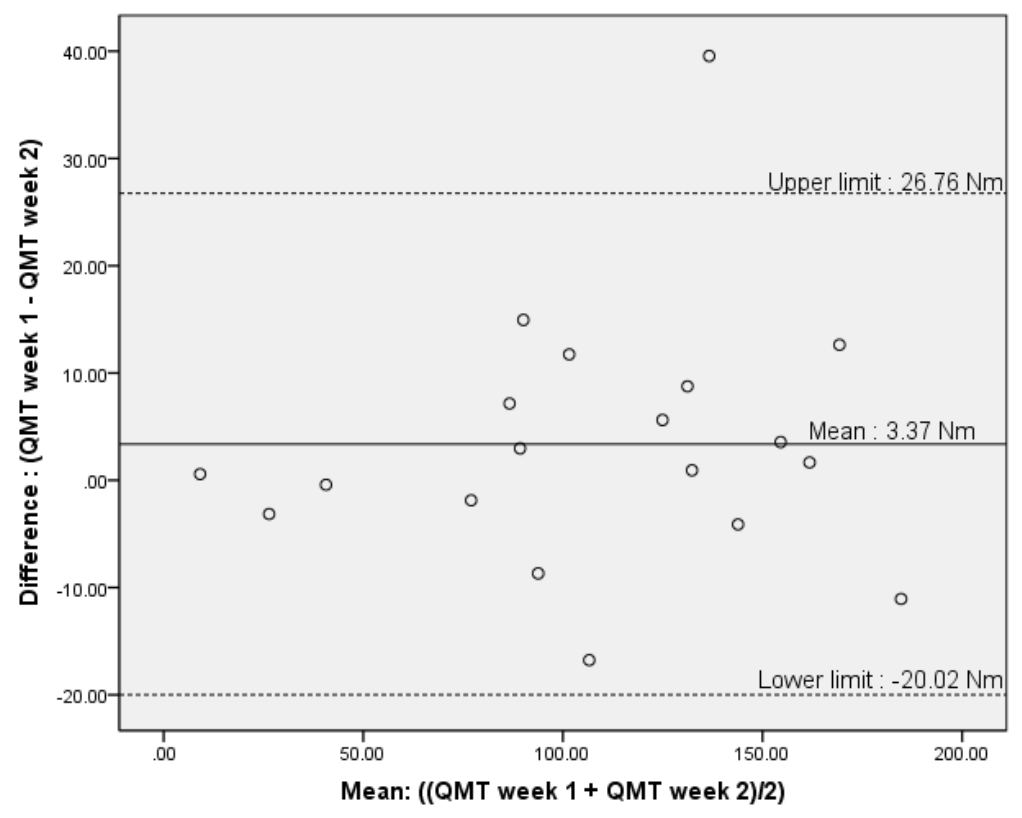

Figure 3

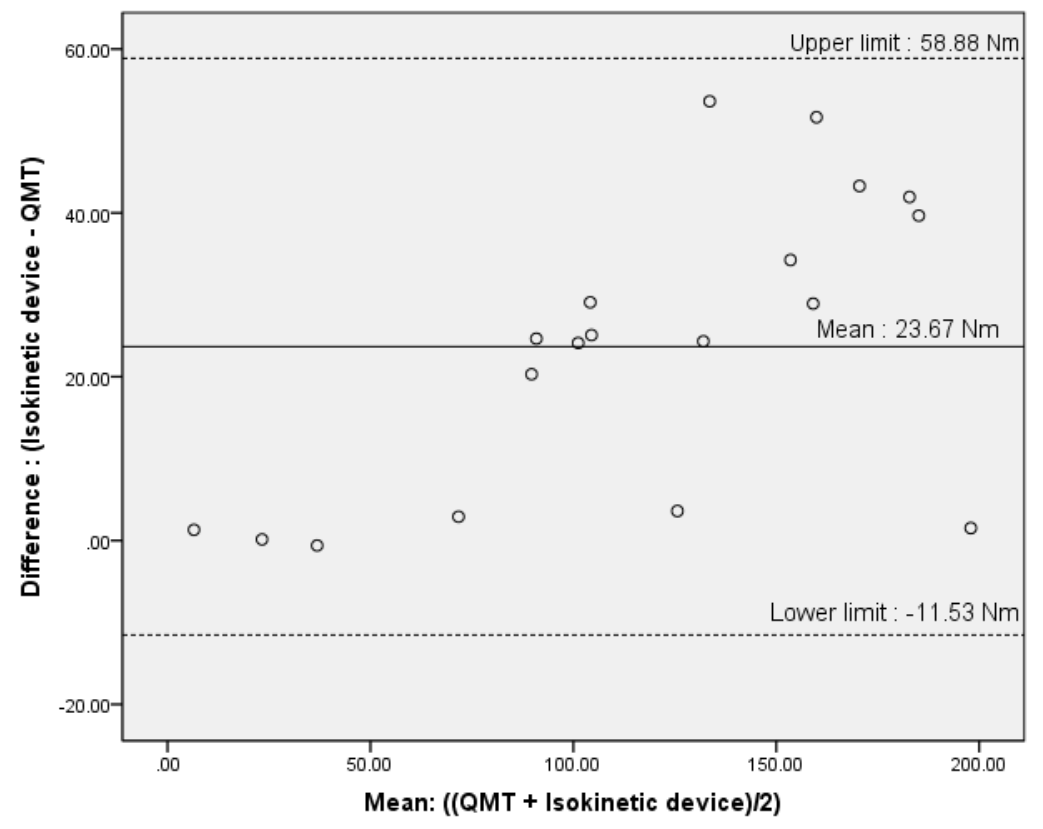

